\title{
Accumulation of Alpha-synuclein Causes Colonic Dysmotility Independently of Enteric Nervous Damage in the Early Stage of Parkinson's Disease
}

(Neurogastroenterol Motil 2012;24:e425-e436)

\section{Yong Sung Kim}

Department of Gastroenterology, Digestive Disease Research Institute, Wonkwang University Sanbon Hospital, Gunpo, Korea

\section{Summary}

Parkinson's disease (PD) is a chronic, progressive neurodegenerative disorder, which is characterized by severe movement impairment. ${ }^{1}$ The main pathologic feature of $\mathrm{PD}$ is a damage of the nigrostriatal dopaminergic pathway and the presence of cyto-plasmic protein aggregates, known as Lewy bodies (LB), in remaining dopaminergic cells. ${ }^{1,2}$ However, pathology of PD was observed in other area including gastrointestinal (GI) tract and constipation is the most common non-motor symptom of PD. ${ }^{1}$ Only a few studies have reported the alteration of enteric nervous system (ENS) related to GI dysfunction in PD. ${ }^{2,3}$

Wang et $\mathrm{al}^{4}$ recently reported age-related alterations in colonic myenteric ganglia and defecation using mice overexpressing wild-type human $\alpha$-synuclein under the Thy-1 promoter (Thy1aSyn). They previously reported that 12 months old mice overexpressing $\alpha$-synuclein exhibited increased colonic transit time and content compared with wild-type. ${ }^{5}$ In current experiment, they investigated whether the onset of colonic motor dysfunction in Thy1-aSyn mice occurred at earlier lifetime. When mice were exposed to novel environment which consisted of placing singly in a new cage without food, Thy1-aSyn mice aged 2.5-3 and 7-8 months exhibited decreased fecal pellet expulsion at 15 min compared to control. While 2.5-3 months old mice did not show significant reduction in the 30-60 minutes, 7-8 months old mice had continuous reduction during 15-60 minutes. These mice subsequently were re-fed during 2 hours and showed significantly decreased fecal pellet output compared to controls in both age. Interestingly, there was no significant change in gastric emptying in Thy1-aSyn mice.

Colonic tyrosine hydroxylase, vasoactive intestinal polypeptide (VIP), peripheral choline acetyltransferase (pChAT) and neuronal nitric oxide synthase (nNOS) immunoreactivity were not significantly different between Thy1-aSyn mice and control. However, the staining for $\alpha$-synuclein was 3 -fold higher in Thy1-aSyn mice compared to control and it was mainly localized to varicosities adjacent to pChAT immunoreactive neuronal fibers. Authors concluded that $\alpha$-synuclein is overproduced in the ENS earlier than the loss of striatal dopamine, and enteric

Received: March 22, 2013 Revised: March 25, 2013 Accepted: March 26, 2013

(c) This is an Open Access article distributed under the terms of the Creative Commons Attribution Non-Commercial License (http://creativecommons. org/licenses/by-nc/3.0) which permits unrestricted non-commercial use, distribution, and reproduction in any medium, provided the original work is properly cited.

*Correspondence: Yong Sung Kim, MD, PhD

Department of Gastroenterology, Wonkwang University Sanbon Hospital, 327, Sanbon-ro, Gunpo, Gyeonggi-do 435-804, Korea

Financial support: None.

Tel: +82-31-390-2975, Fax: +82-31-398-2223, E-mail: wms89@hanmail.net

Conflicts of interest: None. 
neurotransmitters such as dopamine, VIP and nitric oxide may not be important in the colon dysmotility at least in the early stage of PD.

\section{Comment}

PD has not only motor symptoms but also multiple GI symptoms including salivary excess, dysphagia, nausea, delayed gastric emptying, and constipation. ${ }^{6}$ Constipation is the most prominent GI symptom of $\mathrm{PD}$ and their prevalence ranged from $20-89 \%$ in various studies. ${ }^{6}$ Constipation occurs at all stages of $\mathrm{PD}$, even in a preclinical stage. ${ }^{1,6}$ Savica et al ${ }^{7}$ reviewed medical records of 169 subjects who developed PD in Omsted county and investigated the relationship between constipation and PD. They found that constipation precedes the classic motor signs of $\mathrm{PD}$ up to 20 years and suggested that constipation might be an early manifestation of the neurodegenerative process underlying PD. ${ }^{7}$ In spite of their high prevalence, pathophysiology of constipation in $\mathrm{PD}$ has not been investigated in detail and the pathological changes in the ENS of PD still need to be elucidated. ${ }^{1}$

Damage of the ENS in PD is the most plausible explanation for GI dysfunction. So far, it has not been clear whether enteric neurons are damaged, or to what extent they are impaired in PD. ${ }^{1}$ One study using colectomy specimen from patients with PD showed dopaminergic neuronal loss in the colonic myenteric plexus and submucosal plexus, whereas other types of neurons were not affected. ${ }^{8}$ nNOS and VIP immunoreactive neurons in the GI tract also could be affected in PD. ${ }^{9}$ However, study using colonic biopsies from patients with PD showed no definite neuronal loss in the submucosal plexus. ${ }^{10}$

The accumulation of specific pathologic material, such as $\alpha$-synuclein, in the ENS also may cause GI dysfunction in PD. The presence of LB and Lewy neurites has been demonstrated in the ENS of patients with PD in several studies. ${ }^{9,11}$ In the immunohisochemical staining from autopsy specimen of patients with PD, $\alpha$-synuclein immunoreactive aggregations were found in the gastric myenteric and submucosal plexuses. ${ }^{12}$ Lewy neurites immunoreactive for both neurofilament and $\alpha$-synuclein were found in the colonic biopsies and the amount of Lewy neurites in the ENS was positively correlated with constipation. ${ }^{13}$ In current study, Wang et $\mathrm{al}^{4}$ demonstrated that neuronal loss of ENS is not required in dysmotility at least in early stage PD because there was no obvious colonic neuronal loss in the Thy1-aSyn mice. Instead, they suggested that accumulated $\alpha$-synuclein around $\mathrm{pChAT}$ myenteric neurons alters inputs to cholinergic neurons and it may contribute to decreased colonic motility.

Because constipation occurs before motor symptom ${ }^{7}$ and pathologic alterations in the ENS can occur during the early stage of PD even before the central nervous system is affected, ${ }^{8,10-12}$ it was suggested that the GI tract is an early target of $\mathrm{PD}^{14}$ In line with clinical findings, Wang et $\mathrm{al}^{4}$ demonstrated that older group had more reduction in fecal pellet output compared to younger group. This finding indicates progression in the alteration of the defecation response to stress with age in PD.

Because of the difficulty in discovering pathological changes of the ENS in patient with PD and their heterogeneous pathophysiology, appropriate animal models are required in the study of PD. ${ }^{1}$ Current animal models of $\mathrm{PD}$ can be categorized into genetic and neurotoxic models. ${ }^{15}$ Unfortunately, neither of these models exhibited motor symptom, neurodegeneration such as loss of dopamine neuron, and LB accumulation all together. Wang et $\mathrm{al}^{4}$ used mice overexpressing human $\alpha$-synuclein to investigate colonic neuronal change in PD. However, these mice do not have delayed gastric emptying which is highly prevalent in patient with PD. ${ }^{6}$ Although this genetic model was created based on potential mechanism which was identified in PD, it does not exhibit neurodegeneration and phenotypes which are observed in $\mathrm{PD}^{2}$ Toxin models exhibit motor symptom and peripheral neurochemical damage, whereas they have some drawbacks such as the different time factor from human condition. ${ }^{2,916}$ Therefore, it is important to select an appropriate animal model for target symptoms which is the primary interest in the experiment of PD. ${ }^{2}$ The discovery of alteration in the ENS in PD using animal models may help us to find new therapeutic target for GI dysfunction and offer better patient care in this chronic disease.

\section{References}

1. Natale G, Pasquali L, Ruggieri S, Paparelli A, Fornai F. Parkinson's disease and the gut: a well known clinical association in need of an effective cure and explanation. Neurogastroenterol Motil 2008;20: 741-749.

2. Tieu K. A guide to neurotoxic animal models of Parkinson's disease. Cold Spring Harb Perspect Med 2011;1:a009316.

3. Anderson G, Noorian AR, Taylor G, et al. Loss of enteric dopaminergic neurons and associated changes in colon motility in an MPTP mouse model of Parkinsons disease. Exp Neurol 2007;207: 4-12.

4. Wang L, Magen I, Yuan PQ, et al. Mice overexpressing wild-type human alpha-synuclein display alterations in colonic myenteric ganglia and defecation. Neurogastroenterol Motil 2012;24:e425-436.

5. Wang L, Fleming SM, Chesselet MF, Taché Y. Abnormal colonic 
motility in mice overexpressing human wild-type alpha-synuclein. Neuroreport 2008;19:873-876.

6. Pfeiffer RF. Gastrointestinal dysfunction in Parkinson's disease. Parkinsonism Relat Disord 2011;17:10-15.

7. Savica R, Carlin JM, Grossardt BR, et al. Medical records documentation of constipation preceding Parkinson disease: A case-control study. Neurology 2009;73:1752-1758.

8. Singaram C, Ashraf W, Gaumnitz EA, et al. Dopaminergic defect of enteric nervous system in Parkinson's disease patients with chronic constipation. Lancet 1995;346:861-864.

9. Zhu HC, Zhao J, Luo CY, Li QQ. Gastrointestinal dysfunction in a Parkinson's disease rat model and the changes of dopaminergic, nitric oxidergic, and cholinergic neurotransmitters in myenteric plexus. J Mol Neurosci 2012;47:15-25.

10. Lebouvier T, Chaumette T, Damier P, et al. Pathological lesions in colonic biopsies during Parkinson's disease. Gut 2008;57:17411743 .
11. Wakabayashi K, Takahashi H, Takeda S, Ohama E, Ikuta F. Parkinson's disease: the presence of Lewy bodies in Auerbach's and Meissner's plexuses. Acta Neuropathol 1988;76:217-221.

12. Braak H, de Vos RA, Bohl J, Del Tredici K. Gastric $\alpha$-synuclein immunoreactive inclusions in Meissner's and Auerbach's plexuses in cases staged for Parkinson's disease-related brain pathology. Neurosci Lett 2006;396:67-72

13. Lebouvier T, Neunlist M, Bruley des Varannes S, et al. Colonic biopsies to assess the neuropathology of Parkinson's disease and its relationship with symptoms. PLoS One 2010;5:e12728.

14. Hawkes CH, Del Tredici K, Braak H. Parkinson's disease: a dual-hit hypothesis. Neuropathol Appl Neurobiol 2007;33:599-614.

15. Terzioglu M, Galter D. Parkinson's disease: genetic versus toxin-induced rodent models. FEBS J 2008;275:1384-1391.

16. Blesa J, Phani S, Jackson-Lewis V, Przedborski S. Classic and new animal models of Parkinson's disease. J Biomed Biotechnol 2012; 2012:845618. 